\title{
2nd North American Perpendicular Magnetic Recording Conference to Be Held Jointly with 6th Perpendicular Magnetic Recording Conference
}

Following the debut of the North American Perpendicular Magnetic Recording Conference (NAPMRC2002), which was held January 7-9, 2002, in Miami, Fla., the second conference (NAPMRC2003) will be held jointly with the 6th Perpendicular Magnetic Recording Conference (PMRC2003) in Monterey, Calif., January 6-8, 2003. The object of the joint effort is a highly focused review of the latest progress in the development of perpendicular magnetic recording.

The Joint [NA]PMRC will have a singlesession format, with invited presentations forming the body of the conference. A poster session will showcase contributed presentations. The conference is planned as a highly interactive meeting serving as a forum for evaluating the current status of development efforts in perpendicular magnetic recording. Among the major topics to be discussed are recording media and heads, theory and modeling, measurements and characterization, channels and signal processing, and system integration. A special session will be dedicated to the discussion of future and alternative technologies.

Subject to the usual peer-review process, the invited papers will be published in the July 2003 issue of IEEE Transactions on Magnetics.

NAPMRC2003 is organized and cochaired by Sakhrat Khizroev (University of Miami) and Dmitri Litvinov (Seagate Research) in collaboration with PMRC2003 program co-chairs Hiroaki Muraoka (Tohoku University) and Hajime Aoi (Hitachi). Honorary co-chairs for the Joint [NA]PMRC are Stanley Charap (Carnegie Mellon University) and by Sun-ichi Iwasaki (Tohoku Institute of Technology). To ensure broad representation of industry and academia, the conference program is being developed in close collaboration with a 36-member advisory board that includes top leaders from the key academic and industrial organizations worldwide.
The sole sponsor of NAPMRC2003 is the Magnetics Society of the Institute of Electrical and Electronics Engineers. The following organizations and academic research centers have endorsed the conference: the Materials Research Society, the National Storage Industry Consortium, the American Physical Society, the Center for Micromagnetics and Information Technologies at the University of Minnesota, the Center for Materials for Information Technology at the University of Alabama, the Center for Magnetic Recording Research at the University of California-San Diego, the Center for Research on Information Storage Materials at Stanford University, and the Data Storage Systems Center at Carnegie Mellon University.

Additional information about the conference can be accessed at Web site www. napmrc.org.

DMITRI LITVINOV Seagate Technology Co-chair of NAPMRC2003 